of good practice around this important part of specialist community palliative care.

Method The suite of OACC (Outcome Assessment and Complexity Collaborative) measures were introduced to a hospice community team service in the summer of 2015, and by April 2016 we have demonstrated that the average CNS caseload has reduced in size by $28 \%$. CNSs were delivering more short term interventions, or spells of care, to a patient and their family with the aim of resolving particular problems, rather than keeping patients on caseloads for several months. A telephone caseload was initiated and this allowed the CNSs to maintain contact with patients with stable symptoms but with the potential to deteriorate, who would have otherwise been discharged. This allows earlier identification of further needs and an easy re-referral system for the patient or their family when the needs arise. Use of IPOS (Integrated Palliative care Outcome Scale) has defined these spells of care enabling discharge either back to primary care team, other supportive hospice services or to the telephone caseload. CNSs embraced this way of working and saw the benefits which include improved satisfaction and work/life balance.

Conclusion We expect that using spells of care to define caseload size will be a more sustainable way to maintain manageable caseloads in the future. We aim to produce guidelines of good practice in caseload management using the OACC measures.

\section{P-76 USING THE WELLBEING STAR AS AN OUCOME MEASURE IN HOSPICE OUT-PATIENT SERVICES}

Suzy Hudson. The Hospice of St Francis, Berkhamsted, UK

\subsection{6/bmispcare-2017-hospice. 103}

Background Collection of outcome measures within hospice services is challenging due to the varied conditions and diverse trajectories patients follow. Within The Spring Centre there was no standardised outcome measure in place and therefore no consistent validated evaluation of the impact of services on the health and wellbeing of patients.

Aim To assess if the Wellbeing Star is a viable outcome measure tool to evaluate the impact of out-patient services within The Spring Centre on patients' health and wellbeing.

Method Following recommendation from West Hertfordshire Macmillan Wellbeing and Rehabilitation Project and extensive review of literature and similar services, the Wellbeing Star, a validated and well researched measure, was deemed to be the best potential tool for our patients. After funding by Macmillan, formal training was undertaken and Star licences obtained. Wellbeing Stars are completed at first assessment with further reviews at three months and nine months (or as near to these dates as possible). This is an ongoing evaluation.

Results 27 patients' data was analysed.

An increase in all points of the Star was noted.

The greatest improvements were in 'Feeling positive', 'Managing your symptoms', 'Your lifestyle' (59\%, 56\%, 56\%).

The smallest improvements were in 'Money', 'Where you live' $(22 \%, 26 \%)$.

Conclusions Using the Wellbeing Star has been beneficial in embedding a measure within our practice, showing a positive impact of services on patients. Some limitations are evident and timing of reviews is challenging with the demographics of patients using services. We remain undecided on its appropriateness as deterioration due to disease rather than the services not meeting needs is not illustrated. We will continue to collect data for a further nine months and then assess if it adequately meets the needs of hospice out-patient services. The cost of using the Wellbeing Star will also need to be considered.

\section{P-77 AUDIT OF OUTCOME MEASURE USE IN A HOSPICE}

Sara McLintock, Clare Forshaw, Kate Marley. Woodlands Hospice, Liverpool, UK

\subsection{6/bmjspcare-2017-hospice. 104}

Background The Outcome Assessment and Complexity Collaborative (OACC) created a standardised, validated suite of outcome measures for use in palliative care. The key features are the holistic approach, with involvement of the Multidisciplinary Team (MDT) and the patients/families themselves. Our hospice currently uses three outcome measures: the Integrated Palliative care Outcome Scale (iPOS), the Australia-modified Karnofsky Performance Status (AKPS) and Phase of Illness. All three outcome measures are discussed in the weekly multidisciplinary team meetings, both in the in-patient unit (IPU) and the day hospice.

Aim The use of outcome measures was first piloted in the IPU and day hospice in 2012, but there has been no recent audit of their use. Anecdotally the outcome measures are consistently available for review at the MDT meeting but there is not always an available explanation when the iPOS is incomplete. This audit aims to quantify the compliance.

Methods This is a retrospective audit, aiming to capture all patients in a one month period who were admitted to the IPU or who attended the day hospice for assessment. The standards (all with 100\% targets) will include:

- iPOS offered to patients on admission (IPU) or at first assessment (day hospice)

- iPOS offered weekly thereafter - Reason for non-compliance documented when iPOS not completed

- AKPS and Phase of Illness discussed weekly at the MDT meeting (both IPU and day hospice).

- A secondary project will involve documenting baseline scores and changes in scores during admission or time attending the day hospice.

Results Full results awaited.

Conclusion This project encompasses an audit to assess compliance and a secondary project to explore changes in outcome measures during an episode of care. We hope this information will help to further promote the use of outcome measures in clinical practice throughout the hospice.

\section{P-78 PATIENT-REPORTED OUTCOME MEASURES: HOW FREQUENTLY ARE THEY COMPLETED BY PATIENTS IN HOSPICES?}

Jane Gibbins, Melanie Huddart, Kirsty Scott, Deborah Stevens, Carolyn Campbell. Cornwall Hospice Care, Cornwall, UK

\subsection{6/bmispcare-2017-hospice. 105}

Background Patient-Reported Outcome Measures (PROMs) are fundamental in any care setting to determine the perspective of those receiving the care. A recent systematic review 
highlighted that most evidence about the use of PROMs in palliative care populations is in the outpatient oncology setting. There is little evidence about the completion of PROMs in the inpatient palliative care setting. St Christopher's Index of Patient Priorities (SKIPP) 2 is a validated questionnaire exploring symptoms and quality-of-life and given to all patients on their third and tenth day of hospice stay. The completion of one SKIPP on the third day has been used as a PROM as it enquires about the patients' symptoms and quality of life prior to, and after contact/admission to the hospice service.

Methods A retrospective analysis from 150 consecutive admissions lasting four or more days across two hospice sites in the South West of England to determine the completion rates of SKIPP.

Results Of 150 admissions, 61 stayed less than 10 days (and thus expected to complete one SKIPP) and 89 stayed longer than 10 days (and thus expected to complete two SKIPPs). One SKIPP form was completed by $57.3 \%$ of all patients. Of the patients who stayed for 10 days or greater, $33.7 \%$ completed a second SKIPP form. The most common reasons for non-completion were the patient was too unwell $(44.3 \%)$, or the form was not able to be completed on day three $(21.4 \%)$ and therefore deemed an inappropriate outcome measure.

Discussion Nearly $60 \%$ of patients managed to complete one SKIPP questionnaire which can be used alone as a PROM for patients in an inpatient palliative care setting. This suggests PROM can be routinely embedded into clinical practice within a hospice setting.

\section{P-79 REMOVING TECHNICAL BARRIERS: IPOS TIMELINE TOOL AND IPOS REPORT WITH ST BARNABAS HOSPICE AND LOROS}

Sean Leathen. Leathen Healthcare IT Solutions, Bolton, UK

\subsection{6/bmispcare-2017-hospice.106}

Aims Two tools are now in use across St Barnabas and LOROS sites, which support clinicians in getting the best outcomes from IPOS and OACC measures. These are the IPOS Timeline tool and IPOS Report. The IPOS Timeline tool generates a series of graphs about a single patient. The IPOS Report provides a comprehensive assessment of a service's performance for a given time frame. These aim to help services to make the best use of all their IPOS data, by automating processes and removing any technical barriers.

Results The IPOS Timeline has been designed for clinicians. By pressing a single button in a SystmOne extract, it creates a set of graphs showing all IPOS information about the patient. Using this, the clinician can see trends, current and past problems at a glance. This saves time with the patient, or in explaining the patient's history to other clinicians. The IPOS Report has automated the task of analysing all IPOS data across an entire organisation. It uses this information to create an in depth report of service performance, and a full suite of graphs and measures for each IPOS indicator. No data analysis or advanced computer skills are required. The IPOS Report shows where teams are performing well and what can be improved. It contains graphs of service trends over time, and visualises the current level of patient care and how patients needs are changing. A summary shows all this at service level, with a simple rating system. Together, these tools have removed the barrier of technical knowledge and analytical skills needed to handle large amounts of IPOS data. This has enabled services to become more responsive and improve their delivery of patient care.

\section{P-80 SCOPING THE LOCAL LANDSCAPE OF END OF LIFE CARE WITH ROUTINE DATA AND QUALITATIVE RESEARCH}

'Lynn Sudbury-Riley, 'Phillipa Hunter-Jones, 'Jonathan Moss, '2ucy Smith. 'University of Liverpool, Liverpool, UK; ${ }^{2}$ Oakhaven Hospice, Lymington, UK

\subsection{6/bmjspcare-2017-hospice.107}

Background Hospices need to engage in research to ensure provision of the highest possible quality of palliative and end of life care for patients and families. A hospice collaborated with researchers at a world-leading university on a research project to scope the current landscape of palliative and end of life care needs within their geographical area. A postdoctoral research fellow was recruited in May 2017 to conduct and deliver the research over a period of six months.

Aim(s) The research aims to identify what is happening in the hospice's catchment area, and establish why these trends are happening.

Methods Routinely collected health and social care data provide an efficient and useful opportunity for evaluating and improving palliative and end of life care services (Davies et al., 2016). Data from ONS, Public Health England and the primary care network will be used to establish:

- How many people have died in the hospice's catchment area since 2014

- What these people died from

- How many of these deaths did and did not have hospice involvement.

Empirical data collected via qualitative and quantitative research tools will then be used to understand why these trends are happening. Data will be collected from multiple stakeholders and then subjected to systematic thematic analysis. Stakeholders include:

- Health care professionals $(n=20)$

- Hospice service users $(n=20)$

- Non-hospice users $(n=20)$

- Wider population (questionnaires accessed via GP practices).

Results Once the research is complete, these findings will inform the hospice's clinical strategy by providing robust evidence of where direction, activity and resources are most needed. It is anticipated the project will also generate future opportunities for the hospice and research team to develop practical and academic outputs.

Conclusions Hospices and universities can work in partnership to learn about the landscape of end of life care needs in different localities using routine data and qualitative research.

\section{P-81 LEARNING TO FLY: EXPERIENCES OF A FLEDGLING RESEARCH HOSPICE}

Helen Birch. Queenscourt Hospice, Southport, UK

10.1136/bmjspcare-2017-hospice. 108 